\title{
Recognition memory for common words as a function of target/distractor ratio*
}

\author{
P. D. McCORMACK $\dagger$ \\ Carleton University, Ottawa, Canada KIS 5B6
}

\begin{abstract}
The Ss were given three recognition-memory lists having target/distractor (T/D) ratios of $3: 1,1: 1$, and $1: 3$. Hit probabilities were invariant across successive fifths of a list under all conditions. They were also inversely related to $\mathrm{T} / \mathrm{D}$ ratio. False alarms increased as a function of fifths of a list, the rate of increase being inversely related to $\mathrm{T} / \mathrm{D}$ ratio. These data were interpreted within an interference framework, hits presumably reflecting the degree to which targets have been interfered with during storage, and false alarms being indicative of test-phase interference.
\end{abstract}

In an earlier study (McCormack \& Swenson, 1972), two groups of Ss, one exposed to rare and the other to common words, were given recognition memory lists consisting of 21 targets followed by a test phase consisting of 21 targets and 21 distractors in random order. The hit rate for rare words was reliably higher than that for common words by as early as the end of the first sixth of the test phase. Beyond that point, hits for both groups had reached a steady state. These two classes of items, therefore, were likely differentially interfered with prior to the test phase; that is, during target storage. The false-alarm data, on the other hand, failed to reflect any group differences during the early part of the test phase. Performance at that point was optimal for both classes of items, and it was not until later on in the list that common words exhibited more false alarms than rare words. For both sets of items, however, false alarms increased systematically across sixths of a list. These false-alarm data, therefore, are consistent with the notion of a second interference locus whereby interference accumulates during the test phase of the list. Increased exposure to distractors is likely the major contributing factor, common words being presumably more susceptible to this type of interference than rare ones.

In the present investigation, recognition memory for common words was examined across fifths of a list under each of three target/distractor (T/D). ratio conditions. In all three conditions, 30 words were presented during the study portion of the list and 40 during the test phase. The $\mathrm{T} / \mathrm{D}$ ratios during the test portion were $3: 1,1: 1$, and $1: 3$. Since the amount of storage interference should be equivalent for all three conditions, it was expected that there would be no reliable differences among them in hit rate and that hits would be invariant across fifths of a list. On the other hand, the three treatments should generate different

\footnotetext{
*Support for this research was received under National Research Council of Canada Grant APA-78. The author is indebted to Stephen Sunter for data collection and to Lydia Little for computer programming.

†Requests for reprints should be sent to P. D. McCormack, Department of Psychology, Carleton University, Ottawa, Ontario, Canada K1S 5B6.
}

false-alarm rates. Condition 1:3 involves the greatest exposure to distractors, while Condition $3: 1$ involves the least. If distractor density is the major contributor to test-phase interference, then it would be expected that the slope of the function relating false-alarm probabilities to fifths of a list should be inversely related to T/D ratio; that is, false alarms should accumulate most rapidly under Condition 1:3 and least rapidly under Condition 3:1, with Condition 1:1 falling somewhere in between.

The decision to examine the data of the earlier investigation by sixths of a list was made after the experiment had been designed and the data collected. The design was such that the T/D ratio was rarely $1: 1$ for any given $\mathrm{S}$ in any given sixth, since the only constraint was that the ratio be $1: 1$ over the whole list. Another purpose of the present study, therefore, was to attempt to reproduce the earlier findings of hit invariance and increasing false alarms within a list in a 1:1 condition under better controlled circumstances.

\section{METHOD}

\section{Subjects}

The Ss were 35 female and 31 male adults who were assigned to one of six treatment sequences in order of their appearance in the laboratory such that the number of Ss per sequence was equal after every sixth S. Most were Carleton University undergraduate students enrolled in summer school. They were paid $\$ 2$ each for participating.

\begin{abstract}
Design
The word pool consisted of 150 common words selected from the 168 item s of the earlier study. For Condition $3: 1,30$ targets were selected at random and without replacement for the study portion of the list. Ten distractors were similarly selected, the 30 targets and 10 distractors appearing at random for the test portion, with the constraint that each fif th of a list consist of six targets and two distractors. The selection procedure was similar for Conditions 1:1 and 1:3. Condition 1:1 consisted of 30 targets, then 20 targets and 20 distractors with four of each class being presented in each fifth. For Condition 1:3, there were 30 targets, then 10 targets and 30 distractors, with two of the former and six of the latter appearing in every fifth. Each $S$ experienced all three treatments, $11 \mathrm{Ss}$ being assigned to each of the six treatment sequences.
\end{abstract}




\section{Apparatus and Procedure}

All materials were presented by means of $35-\mathrm{mm}$ slides mounted in trays in a projector which was located behind the display panel at a distance of $1.4 \mathrm{~m}$. The $\mathrm{S}$ was located $46 \mathrm{~cm}$ in front of the panel. Items appeared at eye level on a $28 \times 23 \mathrm{~cm}$ surface. A moveable $14 \times 10 \mathrm{~cm}$ response unit was positioned horizontally on a desk between $\mathrm{S}$ and the panel. On this unit were mounted two pushbuttons, $5.7 \mathrm{~cm}$ apart, the left button labeled "No" and the right button labeled "Yes." The E was located between $S$ and the projector. The E's panel consisted of two timers, one latency recording device, a switch which allowed for a timer or a manually controlled operation of the projector, as well as a separate switch for manual projection.

The $\mathrm{S}$ was told that he would receive three lists in which 30 words would appear in rapid succession followed by 40 others, some old and some new, at which time he would be required to indicate which were old and which were new. The use of the response unit was then demonstrated. The button labeled "Yes" was to be pressed should $S$ feel that the item was old, whereas the button labeled "No" was to be pressed should S feel that the item was new. He was told to place the index finger of his preferred hand between the two buttons until an item appeared and was instructed to respond as quickly as possible once he had made his decision.

Following this initial instruction period, $\mathrm{S}$ was given a single practice list with nine two-digit numbers serving as targets and appearing consecutively under control of the timers, which were set at $3 \mathrm{sec}$. The $\mathrm{S}$ was told that this task would be more difficult than the one involving words. Following the presentation of the ninth target, a blank slide appeared, during which time $S$ began counting backwards as fast as he could by threes from a three-digit number, which was presented aurally by E. Data from the earlier study are available which indicate that such a procedure is necessary in order to make the main task of sufficient difficulty. Following $75 \mathrm{sec}$ of backward counting, the nine targets and nine distractors were presented in a manual mode and in random order, with $S$ pressing one of the two buttons each time an item appeared. This enabled $\mathrm{E}$ to record S's response as well as its latency.

When $\mathrm{E}$ was assured that $\mathrm{S}$ understood the task, the three lists were presented, with a pause of approximately $1 \mathrm{~min}$ between lists. For this phase of the experiment, the timers were set at $1.4 \mathrm{sec}$ for the presentation of the 30 targets; otherwise, the procedure was identical to that of the practice phase.

\section{RESULTS AND DISCUSSION}

Performance was examined across successive fifths of a list for each of the three T/D ratio conditions. The major dependent variables were hits and false alarms. Hit and correct-rejection reaction times were also examined, but these data will not be reported here. Analysis of variance procedures were employed in order to assess the main effects of conditions and fifths, as well as their interaction. A treatment-sequence main effect was also examined, and in no instance was it found to be statistically reliable. To simplify the hit and false-alarm analyses, the number of hits emitted by $\mathrm{S}$ in any given fifth of a list was multiplied by 1.5 for Condition $1: 1$ and by 3.0 for Condition 1:3. False alarms were similarly transformed by a factor of 1.5 for Condition 1:1 and 3.0 for Condition 3:1.

The earlier finding of hit invariance within a list in a $1: 1$ condition was obtained once more, but this time in a situation where the experimental design was more sophisticated and where the statistical test was more powerful. The phenomenon, therefore, appears to be a reliable one, one which indicates that hits are relatively insensitive to test-phase interference. No reliable Conditions by Fifths interaction was observed in the hit data. There were, however, statistically dependable differences among the three conditions, $F(2,910)=6.16$, $\mathrm{p}<.005$, hits being inversely related to T/D ratio. This finding could, however, be the result of the adoption of different criteria associated with the three conditions, since the $\beta$-values were $1.53,1.00$, and 0.88 for Conditions $3: 1,1: 1$, and $1: 3$, respectively. The $\beta$ s werë all significantly different when compared pairwise. These hit data, therefore, when corrected for criterion differences, are not necessarily inconsistent with the storage interference hypothesis. It might well be that the amount of interference is the same under all three conditions, the differential hit rates merely indicating that $\mathrm{S}$ is more willing to say "yes," and therefore more likely to achieve a hit, under low than under high T/D ratio conditions.

In the earlier study, where a T/D ratio of $1: 1$ was employed, false alarms increased systematically within a list. This finding was observed once more in the present investigation, and under much better controlled conditions. This phenomenon, therefore, appears to reflect the increasing sensitivity of false alarms to test-phase interference as more and more distractors are encountered. When the false-alarm data of all three conditions were examined, the main effect of fifths was found to be statistically dependable, $F(4,910)=6.28$, $p<.01$. This increase in false alarms over fifths, therefore, appears to be characteristic of all three T/D ratio treatments, as would be expected if false alarms reflect test-phase interference. Although the Fifths by Conditions interaction failed to reach an acceptable level of significance, $F(8,910)=1.45, p>.10$, the data were generally in accord with the expectation that the slopes of the functions relating false alarms to fifths would be inversely related to T/D ratio. Although the predicted interaction was nonsignificant, there was a statistically reliable main effect for conditions, $F(2,910)=17.37$, $\mathrm{p}<.001$, Condition 3:1 exhibiting a lower false-alarm rate than either of the other two. This finding is certainly consistent with the prediction that false alarms will be least pronounced when $\mathrm{S}$ is given minimal exposure to distractors. It is also consistent, however, with the $\beta$ differences which exist among the three conditions, and it is impossible to decide between these two alternative accounts on the basis of the present data.

\section{REFERENCE}

McCormack, P. D., \& Swenson, A. L. Recognition memory for common and rare words. Journal of Experimental Psychology, $1972,95,72-77$.

(Received for publication March 13, 1974.) 